\title{
A Gray Code for cross-bifix-free sets
}

\author{
A. Bernini* \\ S. Bilotta* \\ R. Pinzani* \\ V. Vajnovszki ${ }^{\dagger}$
}

January 16, 2020

\begin{abstract}
A cross-bifix-free set of words is a set in which no prefix of any length of any word is the suffix of any other word in the set. A construction of cross-bifix-free sets has recently been proposed by Chee et al. in 2013 within a constant factor of optimality. We propose a trace partitioned Gray code for these cross-bifix-free sets and a CAT algorithm generating it.
\end{abstract}

\section{Introduction}

A cross-bifix-free set of words is a set where, given any two words over an alphabet, possibly the same, any prefix of the first one is not a suffix of the second one and vice versa. Cross-bifix-free sets are involved in the study of distributed sequences for frame synchronization [11. The problem of determining such sets is also related to several other scientific applications, for instance in pattern matching [6] and automata theory [3].

Fixed the cardinality $q$ of the alphabet and the length $n$ of the words, a matter is the construction of a cross-bifix-free set with the cardinality as large as possible. An interesting method has been proposed in [1] for words over a binary alphabet. In a recent paper [5] the authors revisit the construction of [1] and generalize it obtaining cross-bifix-free sets of words with greater cardinality over an alphabet of arbitrary size. They also show that their cross-bifix-free sets have a cardinality close to the maximum possible; and to our knowledge this is the best result in literature about the size of cross-bifix-free sets.

It is worth to mention that an intermediate step between the original method [1] and its generalization in [5] has been proposed in [4]: it is constituted by a different construction of binary cross-bifix-free sets based on

\footnotetext{
*Dipartimento di Matematica e Informatica "U. Dini", Università degli Studi di Firenze, Viale G.B. Morgagni 65, 50134 Firenze, Italy. antonio.bernini@unifi.it, stefano.bilotta@unifi.it, renzo.pinzani@unifi.it

${ }^{\dagger}$ LE2I, Université de Bourgogne, BP 47 870, 21078 Dijon Cedex, France vvajnov@u-bourgogne.fr
} 
lattice paths which allows to obtain greater cardinality if compared to the ones in [1].

Once a class of objects is defined, in our case words, often it could be useful to list or generate them according to a particular criterion. A special way to do this is their generation in a way such that any two consecutive words differ as little as possible, i.e., in Gray code order [8]. In the case the objects are words, as in our, we can specialize the concept of Gray code saying that it is an infinite set of word-lists with unbounded wordlength such that the Hamming distance between any two adjacent words is bounded independently of the word-length [18] (the Hamming distance is the number of positions in which the two successive words differ [9]). Gray codes find useful applications in circuit testing, signal encoding, data compression, telegraphy, error correction in digital communication and others. They are also widely studied in the context of combinatorial objects as: permutations [10, Motzkin and Schröder words [16], derangements [2], involutions [17, compositions, combinations, set-partitions [12, 14, and so on.

In this work we propose a Gray code for the cross-bifix-free set $S_{n, q}^{(k)}$ defined in [5]. It is formed by length $n$ words over the $q$-ary alphabet $A=$ $\{0,1, \ldots, q-1\}$ containing a particular sub-word avoiding $k$ consecutive 0 's (for more details see the next section). First we propose a Gray code for $S_{n, 2}^{(k)}$ over the binary alphabet $\{0,1\}$, then we expand each binary word to the alphabet $A$. The expansion of a binary word $\alpha$ is obtained replacing all the 1 's with the symbols of $A$ different from 0 producing a set of words with the same trace $\alpha$. The Gray code we get is trace partitioned in the sense that all the words with the same trace are consecutive.

\section{Definitions and tools}

Let $n \geq 3, q \geq 2$ and $1 \leq k \leq n-2$. The cross-bifix-free set $S_{n, q}^{(k)}$ defined in [5] is the set of all length $n$ words $s_{1} s_{2} \cdots s_{n}$ over the alphabet $\{0, \ldots, q-1\}$ satisfying:

- $s_{1}=\cdots=s_{k}=0$;

- $s_{k+1} \neq 0$;

- $s_{n} \neq 0$;

- the subword $s_{k+2} \ldots s_{n-1}$ does not contain $k$ consecutive 0's.

Throughout this paper we are going to use several standard notations which are typical in the framework of sets and lists of words. For the sake of clearness we summarize the ones used here.

For a set of words $L$ over an alphabet $A$ we denote by $\mathcal{L}$ an ordered list for $L$, and 
- $\overline{\mathcal{L}}$ denotes the list obtained by covering $\mathcal{L}$ in reverse order;

- if $\mathcal{L}^{\prime}$ is another list, then $\mathcal{L} \circ \mathcal{L}^{\prime}$ is the concatenation of the two lists, obtained by appending the words of $\mathcal{L}^{\prime}$ after those of $\mathcal{L}$;

- first $(\mathcal{L})$ and last $(\mathcal{L})$ are the first and the last word of $\mathcal{L}$, respectively;

- if $u$ is a word in $A^{*}$, then $u \cdot \mathcal{L}($ resp. $\mathcal{L} \cdot u$ ) is a new list where each word has the form $u \omega$ (resp. $\omega u$ ) where $\omega$ is any word of $\mathcal{L}$;

- if $u$ is a word in $A^{*}$, then $|u|$ is its length, and $u^{n}=\underbrace{u u u \ldots u}_{n}$.

For our purpose we need a Gray code list for the set of words of a certain length over the $(q-1)$-ary alphabet $\{1,2, \ldots, q-1\}, q \geq 3$. An obvious generalization of the Binary Reflected Gray Code [8] to the alphabet $\{1,2, \ldots, q-1\}$ is the list $\mathcal{G}_{n, q}$ for the set of words $\{1,2, \ldots, q-1\}^{n}$ defined in [7, 19] where is also shown that it is a Gray code with Hamming distance 1 . The authors defined this list as:

$$
\mathcal{G}_{n, q}=\left\{\begin{array}{cc}
\lambda & \text { if } n=0, \\
1 \cdot \mathcal{G}_{n-1, q} \circ 2 \cdot \overline{\mathcal{G}_{n-1, q}} \circ \cdots \circ(q-1) \cdot \mathcal{G}_{n-1, q}^{\prime} & \text { if } n>0,
\end{array}\right.
$$

where $\mathcal{G}_{n-1, q}^{\prime}$ is $\mathcal{G}_{n-1, q}$ or $\overline{\mathcal{G}_{n-1, q}}$ according on whether $q$ is even or odd. The reader can easily verify the following proposition.

Proposition 2.1. For $q \geq 3$,

- $\operatorname{first}\left(\mathcal{G}_{n, q}\right)=1^{n}$;

- last $\left(\mathcal{G}_{n, q}\right)=(q-1) 1^{n-1}$ if $q$ is odd, and $(q-1)^{n}$ if $q$ is even.

Now we are going to present another tool we need in the paper. If $\beta$ is a binary word of length $n$ such that $|\beta|_{1}=t$ (the number of 1's in $\beta$ ), we define the expansion of $\beta$, denoted by $\epsilon(\beta)$, as the list of $(q-1)^{t}$ words, where the $i$-th word is obtained by replacing the $t$ 1's of $\beta$ by the $t$ symbols (read from left to right) of the $i$-th word in $\mathcal{G}_{t, q}$. For example, if $q=3$ and $\beta=01011$ (the trace), then $\mathcal{G}_{3,3}=(111,112,122,121,221,222,212,211)$ and $\epsilon(\beta)=(01011,01012,01022,01021,02021,02022,02012,02011)$. Notice that in particular first $(\epsilon(\beta))=\beta$ and all the words of $\epsilon(\beta)$ have the same trace.

We observe that $\epsilon(\beta)$ is the list obtained from $\mathcal{G}_{t, q}$ inserting some 0's, each time in the same positions. Since $\mathcal{G}_{t, q}$ is a Gray code and the insertions of the 0's does not change the Hamming distance between two successive word of $\epsilon(\beta)$ (which is 1), the following proposition holds.

Proposition 2.2. For any $q \geq 3$ and binary word $\beta$, the list $\epsilon(\beta)$ is a Gray code. 


\section{Trace partitioned Gray code for $S_{n, q}^{(k)}$}

Our construction of a Gray code for the set $S_{n, q}^{(k)}$ of cross-bifix-free words is based on two other lists:

- $\mathcal{F}_{n}^{(k)}$, a Gray code for the set of binary words of length $n$ avoiding $k$ consecutive 0's, and

- $\mathcal{H}_{n, q}^{(k)}$, a Gray code for the set of $q$-ary words of length $n$ which begin and end by a non zero value and avoiding $k$ consecutive 0 's. In particular, $\mathcal{H}_{n, 2}^{(k)}=1 \cdot \mathcal{F}_{n-2}^{(k)} \cdot 1$.

Finally, we will define the Gray code list $\mathcal{S}_{n, q}^{(k)}$ for the set $S_{n, q}^{(k)}$ as $0^{k} \cdot \mathcal{H}_{n-k, q}^{(k)}$.

\subsection{The list $\mathcal{F}_{n}^{(k)}$}

Let $\mathcal{C}_{n}$ be the list of binary words defined as:

$$
\mathcal{C}_{n}=\left\{\begin{array}{cl}
\lambda & \text { if } n=0, \\
1 \cdot \overline{\mathcal{C}_{n-1}} \circ 0 \cdot \mathcal{C}_{n-1} & \text { if } n \geq 1,
\end{array}\right.
$$

with $\lambda$ the empty word. The list $\mathcal{C}_{n}$ is a Gray code for the set $\{0,1\}^{n}$ and it is a slight modification of the original Binary Reflected Gray Code list defined in 8 .

By the definition of $\mathcal{C}_{n}$ given in relation (2), we have for $n \geq 1$,

- last $\left(\mathcal{C}_{n}\right)=0 \cdot \operatorname{last}\left(\mathcal{C}_{n-1}\right)=0^{n}$;

- $\operatorname{first}\left(\mathcal{C}_{n}\right)=1 \cdot \operatorname{first}\left(\overline{\mathcal{C}_{n-1}}\right)=1 \cdot \operatorname{last}\left(\mathcal{C}_{n-1}\right)=10^{n-1}$.

Let now define the list $\mathcal{F}_{n}^{(k)}$ of length $n$ binary words as:

$$
\mathcal{F}_{n}^{(k)}=\left\{\begin{array}{cr}
\mathcal{C}_{n} & \text { if } 0 \leq n<k, \\
1 \cdot \overline{\mathcal{F}_{n-1}^{(k)}} \circ 01 \cdot \overline{\mathcal{F}_{n-2}^{(k)}} \circ 001 \cdot \overline{\mathcal{F}_{n-3}^{(k)}} \circ \cdots \circ 0^{k-1} 1 \cdot \overline{\mathcal{F}_{n-k}^{(k)}} & \text { if } n \geq k .
\end{array}\right.
$$

For $k \geq 2$ and $n \geq 0, \mathcal{F}_{n}^{(k)}$ is a list for the set of length $n$ binary words with no $k$ consecutive 0's, and Proposition 3.2 says that it is a Gray code (actually, $\mathcal{F}_{n}^{(k)}$ is a adaptation of a similar list defined earlier [15]).

It is easy to see that the number of binary words in $\mathcal{F}_{n}^{(k)}$ is given by $f_{n}^{(k)}$, the well known $k$-Fibonacci integer sequence defined by:

$$
f_{n}^{(k)}=\left\{\begin{array}{cl}
2^{n} & \text { if } 0 \leq n<k, \\
f_{n-1}^{(k)}+f_{n-2}^{(k)}+\cdots+f_{n-k}^{(k)}, & \text { if } n \geq k,
\end{array}\right.
$$


and the words in $\mathcal{F}_{n}^{(k)}$ are said $k$-generalized Fibonacci words. For example, the list $\mathcal{F}_{3}^{(3)}$ for the length 3 binary words avoiding 3 consecutive 0's is

$$
\mathcal{F}_{3}^{(3)}=(100,101,111,110,010,011,001) .
$$

\section{Proposition 3.1 .}

- $\operatorname{first}\left(\mathcal{F}_{n}^{(k)}\right)$ is the length $n$ prefix of the infinite periodic word $\left(10^{k-1} 1\right)\left(10^{k-1} 1\right) \ldots$;

- last $\left(\mathcal{F}_{n}^{(k)}\right)$ is the length $n$ prefix of the infinite periodic word $\left(0^{k-1} 11\right)\left(0^{k-1} 11\right) \ldots$

Proof. For the first point, if $1 \leq n<k$, then $\operatorname{first}\left(\mathcal{F}_{n}^{(k)}\right)=\operatorname{first}\left(\mathcal{C}_{n}\right)=10^{n-1}$; and if $n=k$, then first $\left(\mathcal{F}_{n}^{(k)}\right)=1 \cdot \operatorname{first}\left(\overline{\mathcal{F}_{n-1}^{(k)}}\right)=1 \cdot \operatorname{last}\left(\mathcal{C}_{n-1}\right)=10^{k-1}$, and the statement holds in both cases.

Now, if $n>k$, by the definition of $\mathcal{F}_{n}^{(k)}$ we have

$$
\begin{aligned}
\operatorname{first}\left(\mathcal{F}_{n}^{(k)}\right) & =1 \cdot \operatorname{first}\left(\overline{\mathcal{F}_{n-1}^{(k)}}\right) \\
& =1 \cdot \operatorname{last}\left(\mathcal{F}_{n-1}^{(k)}\right) \\
& =10^{k-1} 1 \cdot \operatorname{last}\left(\overline{\mathcal{F}_{n-k-1}^{(k)}}\right) \\
& =10^{k-1} 1 \cdot \operatorname{first}\left(\mathcal{F}_{n-k-1}^{(k)}\right),
\end{aligned}
$$

and recursion on $n$ completes the proof.

For the second point, if $1 \leq n<k$, then last $\left(\mathcal{F}_{n}^{(k)}\right)=\operatorname{last}\left(\mathcal{C}_{n}\right)=0^{n}$; and if $n=k$, then last $\left(\mathcal{F}_{n}^{(k)}\right)=0^{k-1} 1$, and the statement holds in both cases.

Now, if $n>k$, we have

$$
\begin{aligned}
\operatorname{last}\left(\mathcal{F}_{n}^{(k)}\right) & =0^{k-1} 1 \cdot \operatorname{last}\left(\overline{\mathcal{F}_{n-k}^{(k)}}\right) \\
& =0^{k-1} 1 \cdot \operatorname{first}\left(\mathcal{F}_{n-k}^{(k)}\right),
\end{aligned}
$$

and by the first point of the present proposition, recursion on $n$ completes the proof.

Proposition 3.2. The list $\mathcal{F}_{n}^{(k)}$ is a Gray code where two consecutive strings differ in a single position.

Proof. It is enough to prove that there is a 'smooth' transition between any two consecutive lists in the definition of $\mathcal{F}_{n}^{(k)}$ given in relation (3), that is, for any $\ell, 1 \leq \ell \leq k-1$, the words

$$
\alpha=0^{\ell-1} 1 \cdot \operatorname{last}\left(\overline{\mathcal{F}_{n-\ell}^{(k)}}\right)=0^{\ell-1} 1 \cdot \operatorname{first}\left(\mathcal{F}_{n-\ell}^{(k)}\right)
$$

and

$$
\beta=0^{\ell} 1 \cdot \operatorname{first}\left(\overline{\mathcal{F}_{n-\ell-1}^{(k)}}\right)=0^{\ell} 1 \cdot \operatorname{last}\left(\mathcal{F}_{n-\ell-1}^{(k)}\right)
$$


differ in a single position. By Proposition 3.1,

$$
\alpha=0^{\ell-1} 1 \alpha^{\prime}
$$

and

$$
\beta=0^{\ell} 1 \beta^{\prime}
$$

with $\alpha^{\prime}$ and $\beta^{\prime}$ appropriate length prefixes of $\left(10^{k-1} 1\right)\left(10^{k-1} 1\right) \ldots$ and $\left(0^{k-1} 11\right)\left(0^{k-1} 11\right) \ldots$, and so $\alpha$ and $\beta$ differ only in position $\ell$.

As a by-product of the proof of the previous proposition we have the following remark which is critical in algorithm process used for the generating algorithm in Section 4.2 .

Remark 1. If $\alpha=a_{1} a_{2} \ldots a_{n}$ and $\beta=b_{1} b_{2} \ldots b_{n}$ are two successive words in $\mathcal{F}_{n}^{(k)}$ which differ in position $\ell$, then either $\ell=n$ or $a_{\ell+1}=b_{\ell+1}=1$.

\subsection{The list $\mathcal{H}_{n, q}^{(k)}$}

Let $\mathcal{H}_{n, q}^{(k)}$ be the list defined by:

$$
\mathcal{H}_{n, q}^{(k)}=\epsilon\left(\alpha_{1}\right) \circ \overline{\epsilon\left(\alpha_{2}\right)} \circ \epsilon\left(\alpha_{3}\right) \circ \overline{\epsilon\left(\alpha_{4}\right)} \circ \cdots \circ \epsilon^{\prime}\left(\alpha_{f_{n-2}^{(k)}}\right)
$$

with $\alpha_{i}=1 \phi_{i} 1$ and $\phi_{i}$ is the $i$-th binary word in the list $\mathcal{F}_{n-2}^{(k)}$, and $\epsilon^{\prime}\left(\alpha_{f_{n-2}^{(k)}}\right)$ is $\epsilon\left(\alpha_{f_{n-2}^{(k)}}\right)$ or $\overline{\epsilon\left(\alpha_{f_{n-2}^{(k)}}\right)}$ according on whether $f_{n-2}^{(k)}$ is odd or even.

Clearly, $\mathcal{H}_{n, q}^{(k)}$ is a list for the set of $q$-ary words of length $n$ which begin and end by a non zero value, and with no $k$ consecutive 0 's. In particular, $\mathcal{H}_{n, 2}^{(k)}=1 \cdot \mathcal{F}_{n-2}^{(k)} \cdot 1$.

Proposition 3.3. The list $\mathcal{H}_{n, q}^{(k)}$ is a Gray code.

Proof. From Proposition 2.2 it follows that consecutive words in each list $\epsilon\left(\alpha_{i}\right)$ and $\overline{\epsilon\left(\alpha_{i}\right)}$ differ in a single position (and by +1 or -1 in this position). To prove the statement it is enough to show that, for two consecutive binary words $\phi_{i}$ and $\phi_{i+1}$ in $\mathcal{F}_{n-2}^{(k)}$, both pair of words

- last $\left(\epsilon\left(1 \phi_{i} 1\right)\right)$ and first $\left(\overline{\epsilon\left(1 \phi_{i+1} 1\right)}\right)=\operatorname{last}\left(\epsilon\left(1 \phi_{i+1} 1\right)\right)$, and

- last $\left(\overline{\epsilon\left(1 \phi_{i} 1\right)}\right)=\operatorname{first}\left(\epsilon\left(1 \phi_{i} 1\right)\right)$ and first $\left(\epsilon\left(1 \phi_{i+1} 1\right)\right)$

differ in a single position.

In the first case, by Proposition 2.1, the first symbols of last $\left(\epsilon\left(1 \phi_{i} 1\right)\right)$ and of last $\left(\epsilon\left(1 \phi_{i+1} 1\right)\right)$ are both $(q-1)$, and the other symbols are either 1 if $q$ is odd, or $(q-1)$ if $q$ is even; and since $\phi_{i}$ and $\phi_{i+1}$ differ in a single position, the result holds.

In the second case, first $\left(\epsilon\left(1 \phi_{i} 1\right)\right)=1 \phi_{i} 1$ and first $\left(\epsilon\left(1 \phi_{i+1} 1\right)\right)=1 \phi_{i+1} 1$, and again the result holds. 


\subsection{The list $\mathcal{S}_{n, q}^{(k)}$}

Now we define the list $\mathcal{S}_{n, q}^{(k)}$ as

$$
\mathcal{S}_{n, q}^{(k)}=0^{k} \cdot \mathcal{H}_{n-k, q}^{(k)},
$$

and clearly, $\mathcal{S}_{n, q}^{(k)}$ is a list for the set of cross-bifix-free words $S_{n, q}^{(k)}$. In particular,

$$
\mathcal{S}_{n, 2}^{(k)}=0^{k} 1 \cdot \mathcal{F}_{n-k-2}^{(k)} \cdot 1,
$$

for example, the set $\mathcal{S}_{8,2}^{(3)}$ of length 8 binary cross-bifix-free words which begin by 000 is

$$
\begin{gathered}
\mathcal{S}_{8,2}^{(3)}=0001 \cdot \mathcal{F}_{3}^{(3)} \cdot 1= \\
=(00011001,00011011,00011111,00011101,00010101,00010111,00010011) .
\end{gathered}
$$

A consequence of Proposition 3.3 is the next proposition.

Proposition 3.4. The list $\mathcal{S}_{n, q}^{(k)}$ is a Gray code.

For the sake of clearness, we illustrate the previous construction for the Gray code list $\mathcal{S}_{8,3}^{(3)}$ on the alphabet $A=\{0,1,2\}$. We have:

$$
\begin{aligned}
\mathcal{G}_{3,3}= & (111,112,122,121,221,222,212,211) ; \\
\mathcal{G}_{4,3}= & (1111,1112,1122,1121,1221,1222,1212,1211,2211,2212,2222, \\
& 2221,2121,2122,2112,2111) ; \\
\mathcal{G}_{5,3}= & (11111, \ldots, 12111,22111, \ldots, 21111) ;
\end{aligned}
$$

and

$$
\begin{aligned}
\mathcal{S}_{8,3}^{(3)}= & (00011001,00011002,00012002,00012001,00022001,00022002, \\
& 00021002,00021001,00021011, \ldots, 00011011,00011111, \ldots \\
& \ldots, 00021111,00021101, \ldots, 00011101,00010101,00010102, \\
& 00010202,00010201,00020201,00020202,00020102,00020101, \\
& 00020111, \ldots, 00010111,00010011,00010012,00010022, \\
& 00010021,00020021,00020022,00020012,00020011) .
\end{aligned}
$$

\section{Algorithmic considerations}

In this section we give a generating algorithm for binary words in the list $\mathcal{F}_{n}^{(k)}$ and an algorithm expanding binary words; then, combining them, we obtain a generating algorithm for the list $\mathcal{H}_{n, q}^{(k)}$, and finally prepending 
$0^{k}$ to each word in $\mathcal{H}_{n-k, q}^{(k)}$ the list $\mathcal{S}_{n, q}^{(k)}$ is obtained. The given algorithms are shown to be efficient.

The list $\mathcal{F}_{n}^{(k)}$ defined in (3) has not a straightforward algorithmic implementation, and now we explain how $\mathcal{F}_{n}^{(k)}$ can be defined recursively as the concatenation of at most two lists, then we will give a generating algorithm for it. Let $\mathcal{F}_{n}^{(k)}(u), 0 \leq u \leq k-1$, be the sublist of $\mathcal{F}_{n}^{(k)}$ formed by strings beginning by at most $u 0$ 's. By the definition of $\mathcal{F}_{n}^{(k)}$, it follows that $\mathcal{F}_{n}^{(k)}=\mathcal{F}_{n}^{(k)}(k-1)$, and

$$
\begin{aligned}
\mathcal{F}_{n}^{(k)}(0) & =1 \cdot \overline{\mathcal{F}_{n-1}^{(k)}} \\
& =1 \cdot \overline{\mathcal{F}_{n-1}^{(k)}(k-1)}
\end{aligned}
$$

and for $u>0$,

$$
\begin{aligned}
\mathcal{F}_{n}^{(k)}(u) & =1 \cdot \overline{\mathcal{F}_{n-1}^{(k)}} \circ 01 \cdot \overline{\mathcal{F}_{n-2}^{(k)}} \circ \cdots \circ 0^{u} 1 \cdot \overline{\mathcal{F}_{n-u-1}^{(k)}} \\
& =1 \cdot \overline{\mathcal{F}_{n-1}^{(k)}} \circ 0 \cdot\left(1 \cdot \overline{\mathcal{F}_{n-2}^{(k)}} \circ \cdots \circ 0^{u-1} 1 \cdot \overline{\mathcal{F}_{n-u-1}^{(k)}}\right) \\
& =1 \cdot \overline{\mathcal{F}_{n-1}^{(k)}} \circ 0 \cdot \mathcal{F}_{n-1}^{(k)}(u-1) .
\end{aligned}
$$

By the above considerations we have the following proposition.

Proposition 4.1. Let $k \geq 2,0 \leq u \leq k-1$, and $\mathcal{F}_{n}^{(k)}(u)$ be the list defined as:

$$
\mathcal{F}_{n}^{(k)}(u)=\left\{\begin{array}{ccc}
\lambda & \text { if } & n=0, \\
1 \cdot \overline{\mathcal{F}_{n-1}^{(k)}(k-1)} & \text { if } & n>0 \text { and } u=0, \\
\frac{\overline{\mathcal{F}_{n-1}^{(k)}(k-1)} \circ 0 \cdot \mathcal{F}_{n-1}^{(k)}(u-1)}{} & \text { if } & n, u>0 .
\end{array}\right.
$$

Then $\mathcal{F}_{n}^{(k)}(k-1)$ is the list $\mathcal{F}_{n}^{(k)}$ defined by relation (3).

Now we explain how the relation (5) defining the list $\mathcal{F}_{n}^{(k)}(u)$ can be implemented in a generating algorithm. It is easy to check that $\mathcal{F}_{n}^{(k)}=\mathcal{F}_{n}^{(k)}(k-$ 1) has the following properties: for $\alpha=a_{1} a_{2} \ldots a_{n}$ and $\beta=b_{1} b_{2} \ldots b_{n}$ two consecutive binary words in $\mathcal{F}_{n}^{(k)}$, there is a $p$ such that

- $a_{i}=b_{i}$ for all $i, 1 \leq i \leq n$, except $b_{p}=1-a_{p}$,

- $0^{k-1}$ can not be a suffix of $a_{1} a_{2} \ldots a_{p-1}=b_{1} b_{2} \ldots b_{p-1}$, 
- the sublist of $\mathcal{F}_{n}^{(k)}$ formed by the strings with the prefix $b_{1} b_{2} \ldots b_{p}$ is $b_{1} b_{2} \ldots b_{p} \cdot \mathcal{L}$, where $\mathcal{L}$ is $\mathcal{F}_{n-p}^{(k)}(u-1)$ or $\overline{\mathcal{F}_{n-p}^{(k)}(u-1)}$ according to the prefix $b_{1} b_{2} \ldots b_{p}$ has an even or odd number of 1 's, and $u$ is equal to $k$ minus the length of the maximal 0 suffix of $b_{1} b_{2} \ldots b_{p}$.

Let us consider procedure gen_fib in Figure 1 where process switches the value of $b[p o s]$ (that is, $b[p o s]:=1-b[p o s]$ ), and prints the obtained binary string $b$. By the above remarks and relation (5) in Proposition 4.1 it follows that after the initialization of $b$ by the first string in $\mathcal{F}_{m}^{(k)}$ (given in Proposition 3.1) and printing it out, the call of gen_fib $(1, k-1,0)$ produces the list $\mathcal{F}_{m}^{(k)}$. Moreover, as we will show below, for $m=n-1$ and after the appropriate initialization of $b=b_{1} b_{2} \ldots b_{n}$ the call of gen_fib $(k+2, k-1,0)$ produces the list $0^{k} 1 \cdot \mathcal{F}_{n-k-2}^{(k)} \cdot 1=\mathcal{S}_{n, 2}^{(k)}$.

Procedure gen_fib is an efficient generating procedure. Indeed, each recursive call induced by gen_fib is either

- a terminal call (which does not produce other calls), or

- a call producing two recursive calls, or

- a call producing one recursive call, which in turn is in one of the previous two cases.

By ' $C A T$ ' principle in [13] it follows that procedure gen_fib runs in constant amortised time.

\subsection{Generating $\mathcal{S}_{n, 2}^{(k)}$}

After the initialization of $b_{1} b_{2} \ldots b_{n}$ by $0^{k} 1 \cdot \operatorname{first}\left(\mathcal{F}_{n-k-2}^{(k)}\right) \cdot 1$, with first $\left(\mathcal{F}_{n-k-2}^{(k)}\right)$ given in Proposition 3.1, and printing it out, the call of gen_fib $(k+2, k-$ $1,0)$ where

- $m=n-1$, and

- procedure process called by gen_fib switches the value of $b[p o s]$ (that is, $b[p o s]:=1-b[p o s])$ and prints $b$

produces, in constant amortized time, the list $0^{k} 1 \cdot \mathcal{F}_{n-k-2}^{(k)} \cdot 1=0^{k} \cdot \mathcal{H}_{n-k, 2}^{(k)}$ which is, as mentioned before, the list $\mathcal{S}_{n, 2}^{(k)}$.

\subsection{Generating $\mathcal{S}_{n, q}^{(k)}, q>2$}

Before discussing the expansion algorithm expand needed to produce the list $\mathcal{S}_{n, q}^{(k)}$ when $q>2$ we show that gen_tuple procedure in Figure 2, on which expand is based, is an efficient generating algorithm for the list $\mathcal{G}_{n, q}$ defined in relation (11). Procedure gen_tuple is a 'naive' odometer principle based algorithm, see again [13], and we have the next proposition. 


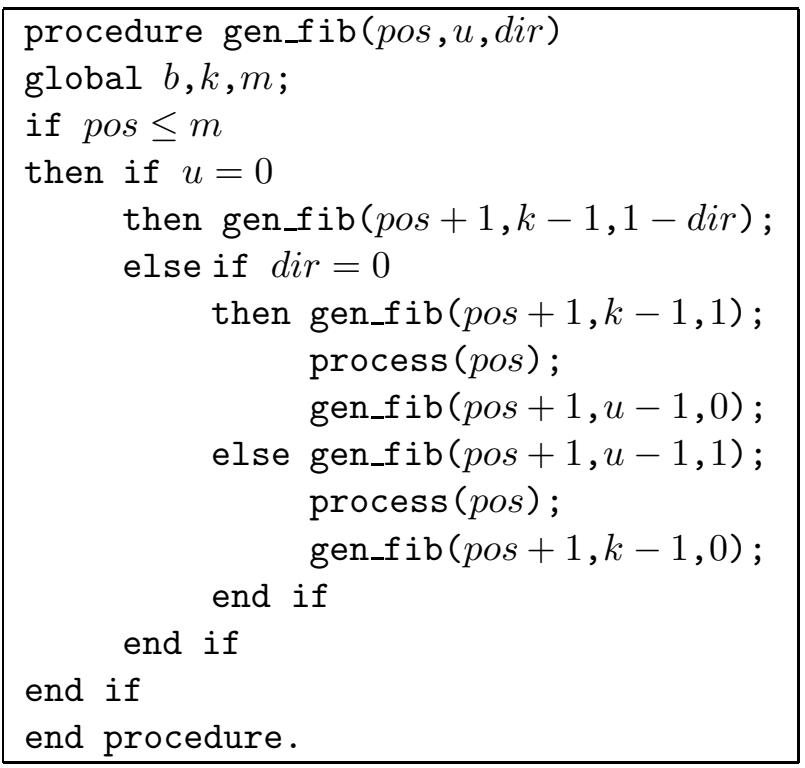

Figure 1: Algorithm producing the list $\mathcal{F}_{n}^{(k)}$ or $\mathcal{S}_{n, q}^{(k)}$, according to the initial values of $m, b$ and the definition of process procedure.

Proposition 4.2. After the initialization of $v$ by $11 \cdots 1$, the first word in $\mathcal{G}_{n, q}$, and $d_{i}$ by 1 , for $1 \leq i \leq n$, procedure gen_tuple produces the list $\mathcal{G}_{n, q}$ in constant amortized time.

Proof. The total amount of computation of gen_tuple is proportional to the number of times the statement $i:=i-1$ is performed in the inner while loop; and for a given $q$ and $n$ let denote by $c_{n}$ this number. So, the average complexity (per generated word) of gen_tuple is $\frac{c_{n}}{q^{n}}$. Clearly, $c_{1}=q-1$ and $c_{n}=(q-1) \cdot n+q \cdot c_{n-1}$, and a simple recursion shows that $c_{n}=q \cdot \frac{q^{n}-1}{q-1}-n$ and finally the average complexity of gen_tuple is $\frac{c_{n}}{q^{n}} \leq \frac{q}{q-1}$.

Now we adapt algorithm gen_tuple in order to obtain procedure expand producing the expansion of a words; and like gen_tuple, procedure expand has a constant average time complexity. More precisely, for a words $b=$ $b_{1} b_{2} \ldots b_{n}$ in $\{0,1, \ldots, q\}^{n}$, with $b_{\ell+1}, b_{n} \neq 0$ let $b^{\prime}$ denote the trace of $b_{\ell+1} b_{\ell+2} \ldots b_{n}$, that is, the word obtained from $b_{\ell+1} b_{\ell+2} \ldots b_{n}$ by replacing each non-zero value by 1 , and $b^{\prime \prime}$ that obtained by erasing each 0 letter in $b_{\ell+1} b_{\ell+2} \ldots b_{n}$. Procedure expand produces the list:

- $b_{1} b_{2} \ldots b_{\ell} \cdot \epsilon\left(b^{\prime}\right)$ if the initial value of $b$ is such that $b^{\prime \prime}$ is the first word in $\mathcal{G}_{\left|b^{\prime \prime}\right|, q}$, or

- $b_{1} b_{2} \ldots b_{\ell} \cdot \overline{\epsilon\left(b^{\prime}\right)}$ if the initial value of $b$ is such that $b^{\prime \prime}$ is the last word in $\mathcal{G}_{\left|b^{\prime \prime}\right|, q}$. 


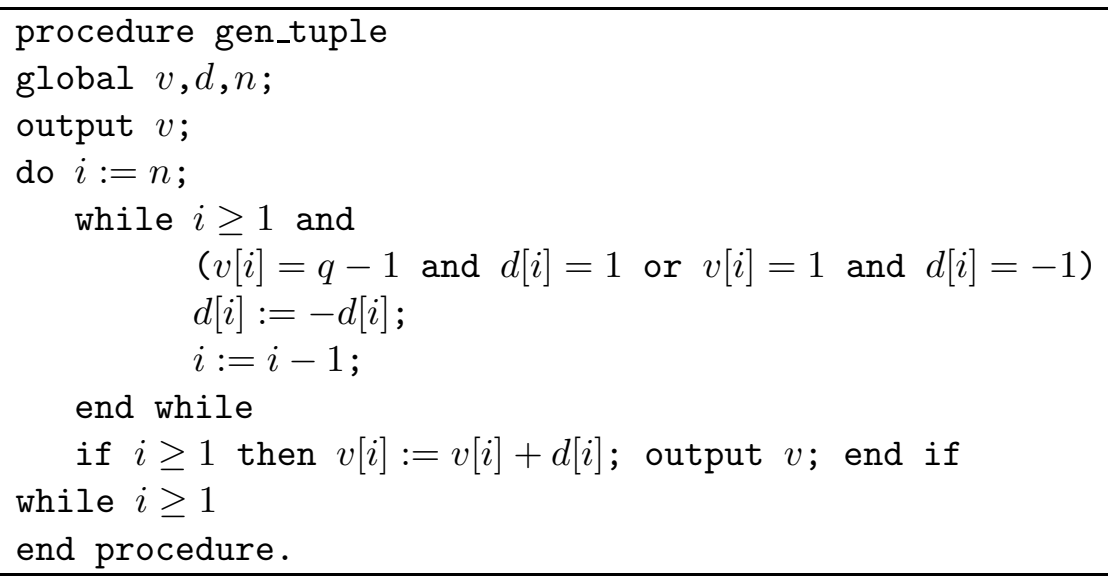

Figure 2: Odometer algorithm producing the list $\mathcal{G}_{n, q}$.

The initial value of $d_{\ell+1}, d_{\ell+2}, \ldots, d_{n}$ are given by: if $b_{i}=1$, then $d_{i}=1$; and if $b_{i}=q-1$, then $d_{i}=-1$; otherwise $d_{i}$ is not defined. In order to access in constant time from a position $i$ in the current word $b$, with $b_{i} \neq 0$, to the previous one, additional data structures are used. The array prec is defined by: if $b_{i} \neq 0$, then prec $_{i}=j$, where $j$ is the rightmost position in $b$, at the left of $i$ and with $b_{j} \neq 0$; and for convenience prec $_{i}=0$ if $i$ is the leftmost non-zero position in $b$.

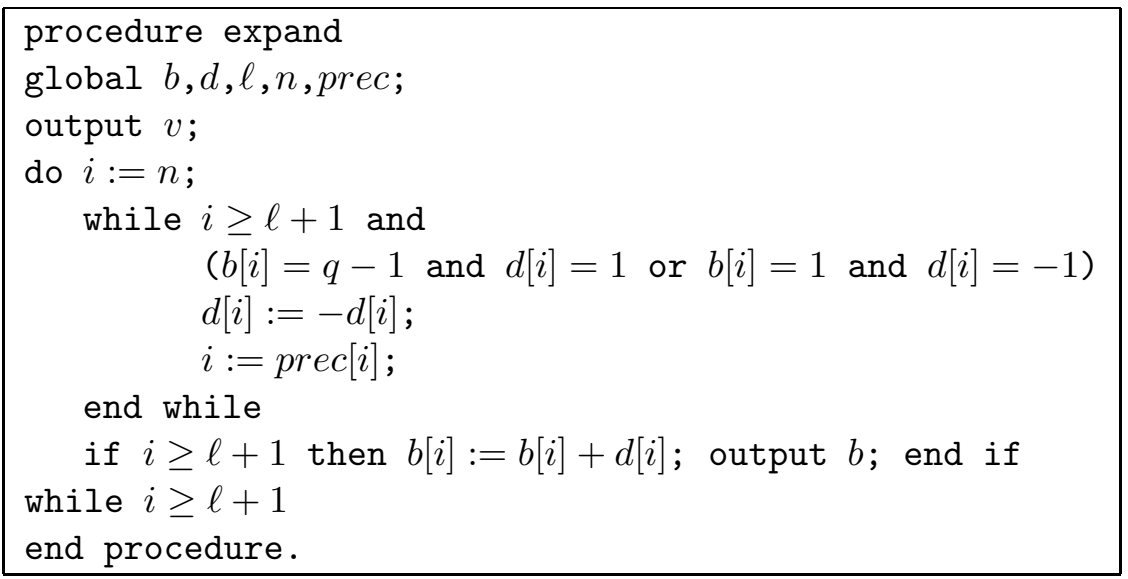

Figure 3: Algorithm expanding a word $b$ and mimicking procedure gen_tuple.

Now we explain procedure process; it calls expand and we will show that when gen_fib in turn calls procedure process in Figure 4, then it produces the list $\mathcal{S}_{n, q}^{(k)}$, with $q>2$. The parameter pos of process is given 


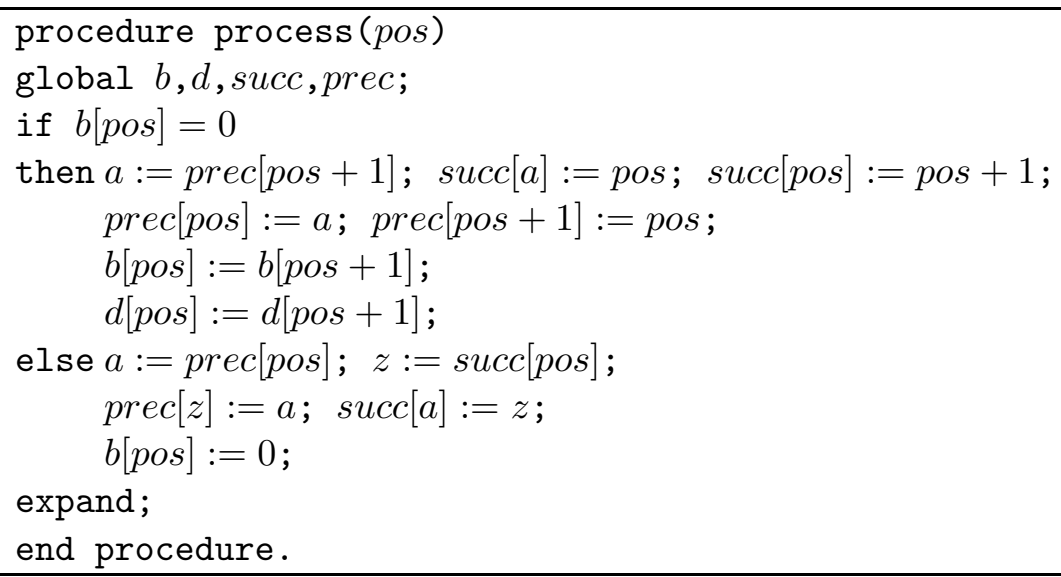

Figure 4: Procedure process called by gen_fib in order to generate the list $\mathcal{S}_{n, q}^{(k)}$.

by the corresponding call of gen_fib, and it gives the position in the current word $b_{1} b_{2} \ldots b_{n}$ in $\mathcal{S}_{n, q}^{(k)}$ where $b_{\text {pos }}$ changes from a non-zero value to 0 , or vice versa. By Remark 1 and the definition of the list $\mathcal{S}_{n, q}^{(k)}$ from $\mathcal{H}_{n-k, q}^{(k)}$, and so from $\mathcal{F}_{n-k-2, q}^{(k)}$, it follows that $b_{\text {pos }+1} \neq 0$. Procedure process, sets $b_{\text {pos }}$ to 0 if previously $b_{\text {pos }} \neq 0$; and sets $b_{\text {pos }}$ to $b_{\text {pos }+1}$ if previously $b_{\text {pos }}=0$, which according to Proposition 2.1, Remark 1 and the definition of the expansion operation is the new value of $b_{\text {pos }}$. In order to access in constant time from a non-zero position in the array $b$ to the previous one, process uses array prec of procedure expand and array succ, defined as: $s u c c_{i}=j$, with $j$ the leftmost position in $b$, at the right of $i$ and with $b_{j} \neq 0$, and succ $_{i}$ is not defined if $i$ is the rightmost non-zero position. In addition, procedure process updates both arrays prec and succ.

For given $q>2, k \geq 2$ and $n \geq k+2$, after the initialization of $b_{1} b_{2} \ldots b_{n}$ by $0^{k} 1$. first $\left(\mathcal{F}_{n-k-2}^{(k)}\right) \cdot 1$, as for generating $\mathcal{S}_{n, 2}^{(k)}$, the call of gen_fib $(k+2, k-$ $1,0)$ where

- $m=n-1$, and

- procedure process is that in Figure 4, and

- procedure expand that in Figure 3, with $\ell=k+1$

produces, in constant amortized time, the list $\mathcal{S}_{n, q}^{(k)}$.

\section{Conclusion and further works}

The cross-bifix-free sets $S_{n, q}^{(k)}$ defined in [5] have the cardinality close to the optimum. They are constituted by particular words $s_{1} s_{2} \ldots s_{n}$ of length 
$n$ over a $q$-ary alphabet. Each word has the form $0^{k} s_{k+1} s_{k+2} \ldots s_{n}$ where $s_{k+1}$ and $s_{n}$ are different from 0 and $s_{k+1} s_{k+2} \ldots s_{n-1}$ does not contain $k$ consecutive 0's. We have provided a Gray code for $S_{n, q}^{(k)}$ by defining a Gray code for the words $s_{k+1} s_{k+2} \ldots s_{n}$ and then prepending the prefix $0^{k}$ to them. Moreover, an efficient generating algorithm for the obtained Gray code is given. We note that this Gray code is trace partitioned in the sense that all the words with the same trace are consecutive. To this aim we used a Gray code for restricted binary strings [15], opportunely replacing the bits 1 with the symbols of the alphabet different from 0 .

A future investigation could be the definition of a Gray code which is prefix partitioned, where all the words with the same prefix are consecutive. Actually, the definition of the sets $S_{n, q}^{(k)}$ shows that it is sufficient to define a prefix partitioned Gray code for the subwords $s_{k+1} s_{k+2} \ldots s_{n}$.

An interesting question arising when one deals with a Gray code $\mathcal{L}$ on a set is the possibility to define it in such a way that the Hamming distance between last $(\mathcal{L})$ and first $(\mathcal{L})$ is 1 (circular Gray code). Usually it is not so easy to have a circular Gray code, unless the elements of the set are not subject to constraints; in our case it is worth to study if the ground-set we are dealing with (which is a cross-bifix free set) allows to find a circular Gray code.

\section{References}

[1] Bajic, D. (2007) On Construction of Cross-Bifix-Free Kernel Sets. 2nd MCM COST 2100, TD(07)237, Lisbon, Portugal.

[2] Baril, J. and Vajnovszki, V. (2004) Gray code for derangements. Discrete Applied Mathematics 140 207-221

[3] Berstel, J., Perrin, D. and Reutenauer, C. (2009) Codes and Automata (Encyclopedia of Mathematics and its Applications). Cambridge University Press.

[4] Bilotta, S., Pergola, E. and Pinzani, R. (2012) A new approach to crossbifix-free sets. IEEE Transactions on Information Theory $\mathbf{5 8}$ 40584063.

[5] Chee, Y. M., Kiah, H. M., Purkayastha, P. and Wang, C. (2013) Crossbifix-free codes within a constant factor of optimality. IEEE Transactions on Information Theory 59 4668-4674.

[6] Crochemore, M., Hancart, C. and Lecroq, T. (2007) Algorithms on strings. Cambridge University Press, Cambridge.

[7] Er, M. C. (1984) On generating the $N$-ary reflected Gray code. IEEE Transaction on Computer 33 739-741. 
[8] Gray, F. (1953) Pulse Code Communication. U.S. Patent 2632058.

[9] Hamming, R. W. (1950) Error detecting and error correcting codes. Bell System Technical Journal 29 147-160.

[10] Johnson, S. M. (1963) Generation of permutations by adjacent transpositions. Mathematics of Computation 17 282-285.

[11] de Lind van Wijngaarden, A. J. and Willink, T. J. (2000) Frame synchronization using distributed sequences. IEEE Transactions on Commununications 48 2127-2138.

[12] Ruskey, F. (1993) Simple combinatorial Gray codes constructed by reversing sublist. Lecture Notes in Computer Science 762 201-208.

[13] Ruskey F. Combinatorial generation, Book in preparation.

[14] Sagan, B. E. (2010) Pattern avoidance in set partitions. Ars Combinatoria 94 (2010) 79-96.

[15] Vajnovszki, V. (2001) A loopless generation of bitstrings without p consecutive ones. Discrete Mathematics and Theoretical Computer Science Springer (2001), 227-240.

[16] Vajnovszki, V. (2001) Gray visiting Motzkin. Acta Informatica 38 793811.

[17] Walsh, T. (2001) Gray codes for involutions. Journal of Combinatorial Mathematics and Combinatorial Computing 36 95-118.

[18] Walsh, T. (2003) Generating Gray Codes in O(1) worst-case time per word. Lecture Notes in Computer Science 2731 73-88.

[19] Williamson, S.G. (1985) Combinatorics for computer science. Computer Science Press, Rockville, Maryland. 